\title{
GESTÃO AMBIENTAL EMPRESARIAL: UM ESTUDO COMPARATIVO DE HOTÉIS EM CANCUN, MÉXICO E VARADERO, CUBA
}

CORPORATE ENVIRONMENTAL MANAGEMENT: A COMPARATIVE STUDY OF HOTELS IN CANCUN, MEXICO, AND VARADERO, CUBA

GESTIÓN AMBIENTAL EMPRESARIAL: UN ESTUDIO COMPARATIVO ENTRE HOTELES DE CANCÚN, MÉXICO Y VARADERO, CUBA

Laureano López Moreda Departamento de Gestión Ambiental, Centro de Servicios Ambientales de Matanzas, Cuba laureano@csam.cu

Doctor en Ciencias Máster en Control de la Contaminación Ambiental Licenciado en Química Elva Esther Vargas Martínez Facultad de Turismo y Gastronomía, Universidad Autónoma del Estado de México elvacolegio@hotmail.com Doctora en Ciencias Ambientales Maestra en Administración Licenciada en Turismo

Data de Submissão: 12-07-2013 Data de Aprovação: 25-03-2014 
Resumo: O presente estudo teve como objetivo comparar sistemas de gestão e certificação ambiental desenvolvido nos destinos turísticos de Varadero, Cuba e Cancun, no México, e sua influência sobre o desempenho do setor hoteleiro. A pesquisa é baseada em revisão da literatura. Foi realizada aplicação de questionários avaliando 31 hotéis e entrevistas em profundidade para os gestores. Os resultados são apresentados por meio de um conjunto de indicadores que avaliam quatro dimensões da gestão ambiental: o consumo de comunicação e responsável, monitoramento ambiental, a disposição para obedecer e ações de gestão ambiental. Embora as amostras estudadas não sejam estatisticamente representativas, a investigação determina que ações sejam implementadas de gestão ambiental em hotéis, reconhecendo comportamentos ambientais de cada um dos grupos estudados. Além de determinar os pontos fortes e fracos em gestão ambiental na indústria hoteleira para ambos os destinos, classificou-se o seu comportamento sob diferentes tipos de manejo.

Palavras-chave: Gestão ambiental. Certificação ambiental. Hotel. Varadero. Cuba. Cancun. México.

Abstract: This study compares the management and environmental certification systems developed in the tourist destinations of Varadero, Cuba and Cancun, Mexico, and their influence on the performance of the hotel industry. The research is based on a literature review, the application of evaluative questionnaires to 31 hotels, and in-depth interviews with the managers of the hotel establishments. The results are presented by means of a set of indicators that assess four dimensions of environmental management: communication and responsible consumption, environmental monitoring, willingness to comply, and environmental management 
actions. Although the studied samples are not statistically representative, the results allowed us to determine what actions companies implement in their environmental management, recognizing the environmental behaviors of each group. Besides helping to determine the strengths and weaknesses in the environmental management of the hotel industry in both destinations, their behavior was classified under different management categories.

Keywords: Environmental management, environmental certification, hotel, Varadero, Cuba, Cancun, Mexico.

Resumen: El presente estudio tuvo como objetivo comparar los sistemas de gestión y certificación ambiental desarrollados en los destinos turísticos de Varadero, Cuba y Cancún, México, y sus resultados en el desempeño del sector hotelero. Se basa en la revisión documental, la aplicación de cuestionarios evaluativos a 31 hoteles y entrevistas en profundidad a directivos de los establecimientos. A pesar de que las muestras estudiadas no son estadísticamente representativas, los resultados permitieron determinar qué acciones de gestión ambiental implementan las empresas, reconociendo así los comportamientos ambientales de cada grupo. Los resultados se presentan desde una propuesta de indicadores que valoran cuatro dimensiones de gestión ambiental: comunicación y consumo responsable, monitoreo ambiental, disposición a cumplir, y acciones de gestión ambiental. Además de contribuir a determinar las fortalezas y debilidades en la gestión ambiental en la industria hotelera de ambos destinos, se calificó su comportamiento en diferentes categorías de manejo.

Palabras clave: Gestión ambiental. Certificación ambiental. Hotelería. Varadero, Cuba. Cancún, México. 
egún los responsables hoteleros reunidos en Roma para el congreso $\bigcirc$ "Turismo Verde y Hoteles", se avizora una verdadera revolución del sector turístico que obligará a los establecimientos hoteleros a realizar ingentes inversiones para captar a un turista cada vez más sensible a la temática ecológica (ANSA, 2012). Es decir, actualmente se vive un nuevo panorama competitivo donde las empresas turísticas buscan modelos no solo rentables, sino también más sostenibles, donde los sistemas de certificación ambiental y las diversas iniciativas voluntarias hacia la ecoeficiencia y la ecoinnovación empresarial juegan un papel determinante (MIRET-PASTOR et al., 2011; KEMP; PEARSON, 2008; ARUNDEL; KEMP, 2009).

Al respecto, los dos sistemas más empleados son la ISO 14001 y el EcoManagement and AuditScheme (EMAS), aunque este último se limita al escenario europeo. No obstante, los gobiernos han desarrollado sistemas de certificación ambiental con carácter voluntario y adecuado a las particularidades de las diferentes naciones. Un ejemplo de estas alternativas lo constituyen los casos de México y Cuba. En México se encuentra el Programa Nacional de Auditoría Ambiental de la Procuraduría Federal de Protección al Ambiente (PROFEPA), mientras en el caso cubano se distinguen dos programas principales: el Aval ambiental y el Reconocimiento Ambiental Nacional del Ministerio de Ciencia, Tecnología y Medio Ambiente (CITMA).

Evaluar el desempeño ambiental en las empresas constituye un imperativo cuando se trabaja por una certificación; la norma cubana NC-ISO 14031 (2005) se refiere a este proceso evaluativo como: "(...) un proceso para facilitar las decisiones de gestión con respecto al desempeño ambiental por medio de indicadores, recopilandoy analizando datos, evaluando información, reportando, comunicando, revisando periódicamente y mejorando este proceso".

La misma norma señala que la Evaluación de Desempeño Ambiental (EDA) integra tres dimensiones de análisis con indicadores propios: a) el comportamiento operacional que refleja los resultados ambientales de una 
entidad, b) los indicadores de gestión que recogen los esfuerzos que realiza la organización en materia ambiental y c) los indicadores de situación ambiental que caracterizan el entorno en que se encuentra enclavada la organización.

Socializar la información ambiental no es solo una demanda o una aspiración de científicos y especialistas en el tema, constituye una necesidad y una contribución a los procesos de mejora que desarrollan las organizaciones empresariales para conocer su posicionamiento respecto a la competencia y a las tendencias más reconocidas en el escenario en el cual se desenvuelven. Sin embargo, diferentes autores (LLULL et al., 2008; PONCE; LABAT, 2007; BONILLA; AVILÉS, 2008; BUENO; SALMADOR; REYES, 2012) reconocen las limitaciones de las empresas hoteleras para comunicar su información ambiental, aspecto que limita el mejoramiento de la gestión ambiental en las empresas del sector.

Anteestaproblemáticasehacepertinenteeldesarrollodenuevasinvestigaciones que faciliten el acceso a información real respecto a la gestión ambiental de las empresas hoteleras. El presente estudio enmarca su aporte científico dentro de los esfuerzos para materializar las EDA en los destinos turísticos en general, y en medir y comparar las acciones de gestión ambiental que las empresas hoteleras mexicanas y cubanas implementan en particular con la finalidad de reducir los impactos ambientales y alcanzar una certificación hacia la sustentabilidad.

Por todo lo anterior, el presente artículo muestra una comparación entre los sistemas nacionales de certificación ambiental vigentes en los destinos turísticos de Cancún, México y Varadero, Cuba; profundizando en su aplicación y en la adopción de prácticas de gestión ambiental en el sector hotelero.

\section{GESTIÓN AMBIENTAL}

Para las empresas hoteleras la gestión ambiental se define como aquella que fomenta un uso eficiente de todos los recursos (energía, agua, materias primas, insumos y equipos en general), y se refleja en una operación más limpia y en servicios cada vez más amigables con el ambiente (DE BURGOS; CÉSPEDES, 2003; GUZMÁN, 2005). 
Las ventajas acerca de la puesta en práctica de acciones de gestión ambiental en las empresas hoteleras son cuestionadas con frecuencia; al respecto Molina et al. (2009, p. 66) argumentan los planteamientos con relación a este debate y señalan que "(...) en el sector hotelero se pueden aplicar medidas de gestión medioambiental como buenas prácticas o implantar Sistemas de Gestión Ambiental que pueden ayudar a reducir costes y a aumentar la diferenciación de un hotel". Señalan además que "los turistas son cada vez más exigentes, lo que fuerza a los hoteleros a adaptarse a sus nuevos gustos y preferencias, incluyendo el mayor respeto hacia el medio ambiente".

La gestión ambiental de las empresas hoteleras se puede visualizar en el marco de innovación tecnológica o más específicamente en el área propia de la actividad innovativa de la empresa, denominada eco-innovación. Para Jänicke (2010) la gestión de la innovación ocupa un lugar central en el modelo ecoeficiente de recursos, considerando que la ecoinnovación se ha convertido en una "dimensión de competencia"; es decir, puede edificarse como un elemento diferenciador de cada empresa con respecto a su competidor; sin embargo, ello está asociado a la eficiencia del proceso y a la rapidez con que se introduzcan los resultados o tecnología. En este sentido el reto impone que tanto los modelos de innovación que se apliquen como sus resultados tengan en cuenta la eficiencia económica de las mejoras introducidas.

Macktola y Jauhari (2007) y Sales, et al. (2010) resaltan que aún no existe un mercado que esté totalmente dispuesto a pagar por servicios ambientalmente compatibles o más sustentables, por tanto los costos de la ecoinnovación no pueden ser rentabilizados mediante la política de precios diferenciados. Por ello, en la hotelería se impone un proceso innovativo con énfasis en la reducción de los costos.

Al respecto, Pelegrín et al. (2010) refieren que el control y mejor aprovechamiento de los recursos naturales en las instalaciones hoteleras está marcando valores agregados importantes en cuanto a los niveles de eficiencia y rentabilidad, lo cual repercute posteriormente en la satisfacción de la clientela y la diferenciación de la competencia.

Las acciones de gestión ambiental más reconocidas dentro de los hoteles son: disminución de residuos sólidos, cantidad y calidad de aguas, consumo de energía y emisiones a la atmósfera (CÉSPEDES; DE BURGOS, 2002). Aunque 
otros autores como López (2011), en su propuesta para hoteles de sol y playa, incluyen además: los residuos peligrosos, el manejo de sustancias químicotóxicas, las emisiones de SAOz, el cuidado de la biodiversidad en el espacio hotelero y la ocupación de la zona costera.

Dentro de las iniciativas aplicadas a la gestión ambiental hotelera sobresalen la adopción de códigos de buenas prácticas ambientales, programas de auditoría, eco-etiquetas, sistemas de certificaciones y otros códigos de auto-regulación. Los hoteles pueden suscribirse también a esquemas que no son dedicados específicamente para el sector, como el Sistema de Gestión Ambiental (SGA) ISO 14001, el Global Compact o la Carta de las empresas para el desarrollo sustentable, concebida por la International Chamber of Commerce. Además, numerosos países han desarrollado diferentes esquemas locales de certificación ambiental.

La tendencia a reportar el desempeño social y ambiental de las empresas ante los diferentes actores sociales 0 partes interesadas ${ }^{1}$, se hace cada vez más creciente. Su logro requiere la estructuración de procedimientos para la evaluación sistemática del desempeño ambiental (SILENIS; HERNÁNDEZ; SILVESTRE; GÓMEZ, 2008).

\section{CARACTERÍSTICAS PRINCIPALES DE LOS DESTINOS TURÍSTICOS CANCÚN, MÉXICO Y VARADERO, CUBA}

\section{CANCÚN, MÉXICO}

En la actualidad Cancún es el destino turístico mexicano con más reconocimiento internacional, se ha convertido en la puerta de entrada a la Riviera Maya, la costa Maya y al Mundo Maya de Centroamérica (FONATUR, 2010). Recibe anualmente más de 3.5 millones de turistas nacionales y extranjeros, principalmente de Estados Unidos, Canadá, Gran Bretaña, Brasil y Argentina, generando una derrama económica por 2,236 millones de dólares en el primer semestre de 2012. Actualmente cuenta con 29,743 habitaciones de hospedaje 1 Resulta oportuno apuntar que al referirse a partes interesadas se incluyen los individuos y otras entidades que aportan valor a la organización, o que de otro modo están interesados en sus actividades o afectados por ellas (NC- ISO 14001, 2005). 
distribuidas en alrededor de 139 hoteles en todas las categorías (BARÓMETRO TURÍSTICO DE CANCÚN, 2012).

El fuerte desarrollo turístico de cuatro décadas ha originado la contaminación visual del paisaje, el desplazamiento de la fauna, generación de residuos y la escasez de agua. (Vargas, 2010). El destino cuenta con nueve plantas de tratamiento de aguas residuales, aunque se estima que anualmente se vierten al manto freático más de 11 millones de metros cúbicos. También derivada del desarrollo turístico intensivo es la gran cantidad de desechos sólidos que deben ser recolectados y tratados en el municipio (IMPLAN, 2007).

Otros problemas asociados al desarrollo del destino son el crecimiento no controlado de la población, por tanto el difícil acceso a la vivienda, la proliferación de asentamientos irregulares y el difícil acceso a servicios básicos (ROMERO, 2009).

Un elemento importante en el entorno ambiental es el relacionado con la ocurrencia de fenómenos meteorológicos extremos como los huracanes Gilberto en 1988 y Wilma en 2005 (VARGAS, 2010). Ambos fenómenos no solo afectaron seriamente la planta hotelera, sino que unidos al efecto negativo de las construcciones sobre las dunas costeras, produjeron la desaparición de la playa, por ello ha sido necesaria la aplicación de proyectos de alimentación artificial de arena que en el 2009 superó los 5 millones de metros cúbicos (ROMERO, 2009).

En la actualidad Cancún constituye un destino de sol y playa, donde impera el "modelo todo incluido", y su futuro, según expertos, estará condicionado a la capacidad de diversificación de la oferta (PAZ, 1995).

VARADERO, CUBA

Varadero es el destino turístico de sol y playa más importante del país (RIVAS et al., 2009). La ciudad turística tiene una superficie de $14.4 \mathrm{~km}^{2}$, de la cual el $58,3 \%$ se encuentra edificada. Hay una intensa actividad de servicios asociada a la actividad turística, así como de pequeña producción. Por otro lado, Varadero ha generado ingresos que significan el 35\% de los beneficios económicos aportados por el turismo al país en años recientes (MOYA et al., 2007). 
Según el sitio Cuba Travel (2011), Varadero viene recibiendo por cuatro años consecutivos más de un millón de turistas, aproximadamente el $40 \%$ de los arribos totales al país, la mayoría procedentes de Canadá, Reino Unido y Alemania, principales mercados emisores del destino. Aparejado a esto hay una explosión del turismo nacional, fundamentalmente en los meses de verano, que hoy constituye el segundo mercado después del canadiense.

La planta hotelera, según información publicada por el Ministerio de Turismo de Cuba (MINTUR) incluye más de 16,700 habitaciones en alrededor de 53 instalaciones hoteleras, el $70 \%$ de las cuales poseen categoría de 4 y 5 estrellas. El $89 \%$ de los hoteles son propiedad absoluta de empresas nacionales, Inmobiliaria del Ministerio de Turismo (70\%) e Inmobiliaria ALMEST S.A. (19\%), mientras el $11 \%$ son de capital mixto. En cuanto a la administración y operación, la misma recae en cuatro cadenas hoteleras nacionales: Gaviota S.A. (10\% de las instalaciones), Cubanacán S.A. (13\%), Gran Caribe (18\%) e Isla Azul (11\%). Estas cadenas han suscrito contratos de administración con cadenas extranjeras en el $64 \%$ de las instalaciones (MOYA et al., 2007; MINTUR, 2012).

Desde el punto de vista ambiental, se incrementa el uso de la playa tanto por turistas extranjeros como por nacionales. Esto provoca la sobreexplotación en algunos tramos, donde se supera la capacidad de carga del ecosistema, y en un mismo lapso se han llegado a identificar picos de más de 60,000 turistas/ día en la península. Otro efecto derivado de la abundante población turística estacional es el aumento en el consumo de agua y electricidad, así como en la generación de residuos (MOYA et al., 2007).

El alcantarillado capta la mayor parte de las aguas residuales producidas por el desarrollo hotelero y la población residente, las cuales son tratadas y dispuestas a la Bahía de Cárdenas. El tratamiento es garantizado por seis plantas municipales que operan bajo las normas establecidas en el país al efecto (LÓPEZ, 2010).

La calidad de aire se ve beneficiada cuando surge la planta ENERGAS, planta que toma los residuales de la extracción petrolera próxima al destino y los convierte en energía eléctrica y en materia prima para otros procesos industriales (MOYA et al., 2007). 
Para los próximos años se anticipa el impulso de Varadero sobre la base de la aplicación científico-técnica de principios de sustentabilidad. La nueva zona de desarrollo se concentra en la sección más septentrional de la península y prevé un crecimiento de hasta 25,000 habitaciones (CABRERA, 2000). El desarrollo futuro de la paya estará signado por la prioridad de su conservación, lo que es, desde ya, una realidad que alcanzará niveles superiores de ejecución con la inclusión de novedosas concepciones tecnológicas y herramientas de manejo costero integrado (MOYA et al., 2007).

\section{COMPARACIÓN ENTRE LOS PROCESOS DE CERTIFICACIÓN AMBIENTAL VIGENTES EN LOS DESTINOS TURÍSTICOS DE CANCÚN, MÉXICO Y VARADERO, CUBA}

El principal sistema de certificación ambiental vigente en México es el Programa Nacional de Auditoría Ambiental de la PROFEPA. El programa promueve desde 1992 la participación voluntaria de los sectores empresariales en el cumplimiento del marco legal y normativo ambiental, así como la adopción de buenas prácticas de operación, con la finalidad de proteger el ambiente y que las empresas mejoren su desempeño en este sentido (PROFEPA, 2009).

Se aplica tanto a empresas del sector primario, comercios y servicios, entre ellos los turísticos. El programa emite los siguientes certificados:

a) Industria limpia. Dirigido al sector manufacturero y de transformación.

b) Calidad Ambiental. Dirigido a organizaciones de comercio y servicio.

c) Calidad Ambiental Turística. Todo tipo de instalaciones con vocación turística: hoteles, campos de golf, áreas naturales protegidas, zoológicos, museos, etc.

En su implementación interviene la Subprocuraduría de Auditoría Ambiental y se rige por la Ley del Equilibrio Ecológico y la Protección del Ambiente en Materia de Autorregulación y Auditorías Ambientales, donde se establecen 
los lineamientos que deberán seguir las empresas interesadas en reconocer y certificar el desempeño ambiental de sus procesos.

El proceso de certificación se articula en tres actos principales, la planeación que incluye la inscripción de la empresa en el registro; la operación, donde se realiza la auditoría y se genera un plan de acción, se firma un compromiso de cumplimiento por parte de la empresa y se da seguimiento al mismo hasta su cumplimiento y finalmente la etapa de certificación. La auditoría incluye diferentes áreas de la gestión ambiental de los establecimientos hoteleros; ellos son: uso y manejo del agua, contaminación del suelo y el subsuelo, contaminación del aire, generación de ruido, generación y manejo de residuos peligrosos, residuos no peligrosos, residuos con manejo especial, riesgo ambiental y atención a emergencias.

El certificado emitido tiene dos años de vigencia, período tras el cual debe ser renovado mediante la presentación de un diagnóstico actualizado de la situación ambiental de la empresa o un reporte de desempeño ambiental para aquellas empresas que además de renovar su certificación evidencien que cuentan con un nivel máximo de desempeño. El reporte debe contener los indicadores, acciones y programas, así como las evidencias necesarias que lo validen (PROFEPA, 2010).

Sin embargo, la acogida de la certificación en el sector turístico no es amplia, según la PROFEPA en el año 2009 las empresas turísticas representaron solo el $4 \%$ del total de certificaciones entregadas en dicho año, mientras que en el 2010 el número de hoteles certificados ascendía a 64 (PROFEPA, 2010).

En Cuba, existen dos esquemas fundamentales que certifican el trabajo ambiental de los hoteles, por una parte se encuentra el Aval Ambiental y por otra el Reconocimiento Ambiental Nacional (RAN).

El RAN es más exigente en cuanto a indicadores y criterios de evaluación. Se rige por la Resolución 27 del Ministerio de Ciencia, Tecnología y Medio Ambiente y prevé dos categorías para su otorgamiento: Reconocimiento Ambiental Nacional a Nivel Básico y Sello Distintivo. En la práctica no es necesario transitar de una categoría a la otra, por tanto se puede obtener directamente el sello 
distintivo. Los requisitos a cumplir para ser certificado un hotel son amplios y abarcan el cumplimiento estricto de la legislación ambiental; la organización de la gestión que incluye su planificación, asignación de responsabilidades y recursos materiales, la incorporación de prácticas de producciones más limpias, el monitoreo ambiental, la introducción de tecnologías ambientales y la gestión ecológicamente racional de más de una veintena de aspectos ambientales. Además aborda cuestiones asociadas a los planes de contingencia, la educación ambiental, la disciplina tecnológica y económico financiera de la empresa, así como los vínculos con otros actores sociales y el gobierno local.

El RAN desde sus inicios resultó un modelo abarcador que tenía en cuenta criterios inherentes a otros sistemas gerenciales como los de seguridad y salud en el trabajo, la gestión económica financiera y la higiene e inocuidad de los alimentos. La distinción es exigente y limitada para muchas empresas hoteleras cubanas, donde la gestión ambiental aún tiene un carácter correctivo. La prioridad la constituye el cumplimiento de la ley para prevenir sanciones o multas. Por otra parte, el mecanismo no contó, ni cuenta, con un proceso adecuado de publicidad que permita el reconocimiento por el mercado nacional e internacional. No obstante, según Terry (2002), el sector turístico representa el $40 \%$ de las empresas que han obtenido o trabajan por el RAN en el país, y en la actualidad esta autora afirma: "El sector turístico es el más representado, aunque actualmente solo son siete los hoteles con tal distinción" (CUBA NOTICIAS, 2011).

Con respecto al Aval ambiental, vigente en Cuba, su obtención es una condicionante a la categorización de los servicios hoteleros, proceso que resulta obligatorio en el país. Se centra en un proceso de planificación estratégica que resulta jurídicamente vinculante, así como su marco de evaluación y concesión son solo a nivel provincial. Además, aunque incluye un proceso de recertificación, el mismo no requiere de la presentación de documentación adicional.

En la tabla 1 se realiza una comparación entre los principales programas de certificación ambiental vigentes en los destinos de Cancún, México y Varadero, Cuba. 
Tabla 1. Comparación entre los modelos de gestión ambiental

\begin{tabular}{|c|c|c|c|}
\hline & $\begin{array}{c}\text { Programa Nacional } \\
\text { de Auditoría } \\
\text { Ambiental (PNAA- } \\
\text { PROFEPA) }\end{array}$ & Aval Ambiental & $\begin{array}{l}\text { Reconocimiento Ambiental } \\
\text { Nacional (RAN) }\end{array}$ \\
\hline Enfoque & $\begin{array}{l}\text { Cumplimiento de la } \\
\text { ley }\end{array}$ & $\begin{array}{c}\text { Categorización } \\
\text { hotelera }\end{array}$ & $\begin{array}{l}\text { Cumplimiento de la ley } \\
\text { y compromisos ante las } \\
\text { autoridades locales y la } \\
\text { comunidad }\end{array}$ \\
\hline $\begin{array}{l}\text { Expresión del } \\
\text { estado deseado } \\
\text { del desempeño }\end{array}$ & Política & $\begin{array}{c}\text { Misión y visión } \\
\text { estratégica }\end{array}$ & Política \\
\hline Carácter & Voluntario & Condicionado & Voluntario \\
\hline $\begin{array}{l}\text { Evaluación } \\
\text { ambiental inicial }\end{array}$ & $\begin{array}{l}\text { Requisito } \\
\text { (Auditoría) }\end{array}$ & $\begin{array}{c}\text { Requisito } \\
\text { (Diagnóstico) }\end{array}$ & Requisito (Diagnóstico) \\
\hline $\begin{array}{l}\text { Criterios a } \\
\text { considerar }\end{array}$ & $\begin{array}{c}\text { Aspectos } \\
\text { Ambientales } \\
\text { Requisitos legales }\end{array}$ & $\begin{array}{l}\text { Requisitos } \\
\text { legales }\end{array}$ & $\begin{array}{c}\text { Aspectos Ambientales } \\
\text { Requisitos legales }\end{array}$ \\
\hline $\begin{array}{l}\text { Instrumento de } \\
\text { planificación }\end{array}$ & Plan de acción & Plan de acción & Programas de mejora \\
\hline $\begin{array}{l}\text { Formación y } \\
\text { capacitación }\end{array}$ & Requisito & Requisito & Requisito \\
\hline $\begin{array}{l}\text { Relaciones con } \\
\text { actores sociales }\end{array}$ & $\begin{array}{l}\text { Solo para la } \\
\text { promoción }\end{array}$ & $\begin{array}{c}\text { No se } \\
\text { considera }\end{array}$ & Requisito de certificación \\
\hline $\begin{array}{c}\text { Documentación } \\
\text { exigida }\end{array}$ & Limitada & Limitada & Amplia \\
\hline $\begin{array}{c}\text { Controles } \\
\text { operacionales }\end{array}$ & $\begin{array}{l}\text { Limitado a las } \\
\text { exigencias de la } \\
\text { legislación }\end{array}$ & $\begin{array}{l}\text { Limitado a las } \\
\text { exigencias de la } \\
\text { legislación }\end{array}$ & $\begin{array}{l}\text { Limitado a las exigencias } \\
\text { de la } \\
\text { legislación }\end{array}$ \\
\hline
\end{tabular}




$\begin{array}{cccc}\begin{array}{c}\text { Enfrentamiento a } \\ \text { catástrofes }\end{array} & \begin{array}{c}\text { Plan de } \\ \text { contingencia }\end{array} & \begin{array}{c}\text { Plan de } \\ \text { contingencia }\end{array} & \begin{array}{c}\text { Plan de contingencia } \\ \text { Verificación y }\end{array} \\ \begin{array}{c}\text { Informes } \\ \text { ambientales }\end{array} & \begin{array}{c}\text { Actualización } \\ \text { del diagnóstico }\end{array} & \begin{array}{c}\text { Actualización del } \\ \text { diagnóstico }\end{array} \\ & \begin{array}{c}\text { Auditorías } \\ \text { bianuales }\end{array} & \begin{array}{c}\text { Inspecciones } \\ \text { externas }\end{array} & \begin{array}{c}\text { Inspecciones externas } \\ \text { bianuales }\end{array}\end{array}$

Seleccionados

Empleo de indicadores por la entidad y exigidos por la legislación

Limitado a las exigencias de la legislación

No

Mejora continua No explicita Marco de aplicación

Forma de visualización

Período de certificación Sello distintivo Calidad Ambiental Turística Aval del nivel provincial Certificado contemplada No explicita Cuba Cuba (local) Seleccionados por la entidad $y$ exigidos por la legislación

México

2 años 2 años

Seguridad y salud en Otros requisitos No considerados
No el trabajo, higiene de considerados los alimentos, disciplina tecnológica y resultados económico financieros.

Fuente: Elaboración propia

La primera diferencia significativa dentro de los procesos de certificación en ambos países es que para el destino Varadero operan dos sistemas, mientras que para Cancún solamente uno. En general existen similitudes importantes entre el RAN y el PNAA, dado su enfoque al cumplimiento de la ley y el compromiso 
social, el carácter netamente voluntario de ambos, las exigencias respecto a la política, la educación ambiental, el enfoque hacia la gestión de aspectos ambientales y al cumplimiento de la legislación, la elaboración y exigencia del cumplimiento de planes de acción derivados de procesos de evaluación preliminar, la prioridad que conceden al enfrentamiento a situaciones de emergencia, el uso de indicadores ambientales, los períodos y procedimientos de recertificación, sus limitaciones en cuanto al aseguramiento de la mejora continua y su alcance nacional.

Sin embargo, se diferencian por el empleo de diferentes enfoques pues el RAN incluye las relaciones con otros actores sociales, los instrumentos de evaluación inicial, la forma de visualización de la certificación y los requerimientos documentales; sin embargo, las diferencias más importantes se asocian a los aspectos ambientales considerados por cada esquema (Tabla 2), así como a la inclusión como parte del RAN de requisitos de seguridad y salud en el trabajo, higiene de los alimentos, disciplina tecnológica y resultados económico-financieros.

Tabla 2. Aspectos ambientales considerados por el PNAA y el RAN en el proceso de evaluación y certificación ambiental de hoteles.

\begin{tabular}{lcc}
\hline Aspectos ambientales & PNAA & RAN \\
& (México) & (Cuba)
\end{tabular}

Vínculo con la comunidad, autoridades y otros actores sociales

Patrimonio histórico cultural

Capacitación ambiental

Innovación

Uso y manejo del agua

Consumo de energía y combustible

Consumo de productos químico tóxicos

Áreas verdes y jardinería

Ocupación de la zona costera

$\begin{array}{ll} & \mathbf{x} \\ \mathbf{X} & \mathbf{x} \\ \mathbf{X} & \mathbf{x} \\ & \mathbf{x} \\ & \mathbf{x} \\ & \mathbf{x}\end{array}$

Contaminación del suelo

$\mathbf{X}$

Contaminación el subsuelo

Contaminación del aire 
Generación de ruido

Generación y manejo de residuos peligrosos

Residuos con manejo especial

Residuos no peligrosos

Riesgo ambiental y atención a emergencias

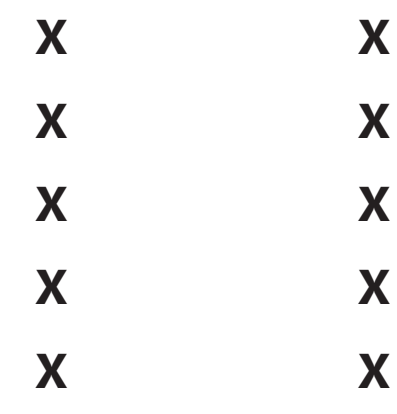

Fuente: Elaboración propia

METODOLOGÍA

En ambos destinos la investigación se realizó entre los años 2009 y 2011. Contaron con un diseño experimental transversal, pues mediante este se explican las condiciones que permanecen en las empresas al recolectar los datos durante un período de tiempo sin manipular las variables que intervienen en la investigación. Mediante la revisión bibliográfica se comparan los procesos de certificación ambiental vigentes en ambos destinos.

Para evaluar la gestión ambiental en los hoteles de Cuba, López (2010) propone un grupo de indicadores agrupados en las categorías de educación, comunicación e información ambiental; control operacional; control gerencial, así como proveedores y consumo responsable. Por su parte, Vargas et al. (2011) en el caso mexicano se basa igualmente en un sistema de indicadores articulados en cuatro dimensiones: acciones de gestión ambiental, comunicación, consumo responsable y monitoreo. Para el análisis de los resultados obtenidos en ambos destinos se asumen las cuatro dimensiones y los indicadores asociados que determinan el comportamiento ambiental de una empresa según Vargas et al. (2011) (ver tabla 3). 
Tabla 3. Criterios para evaluar los resultados de la gestión ambiental en los hoteles.

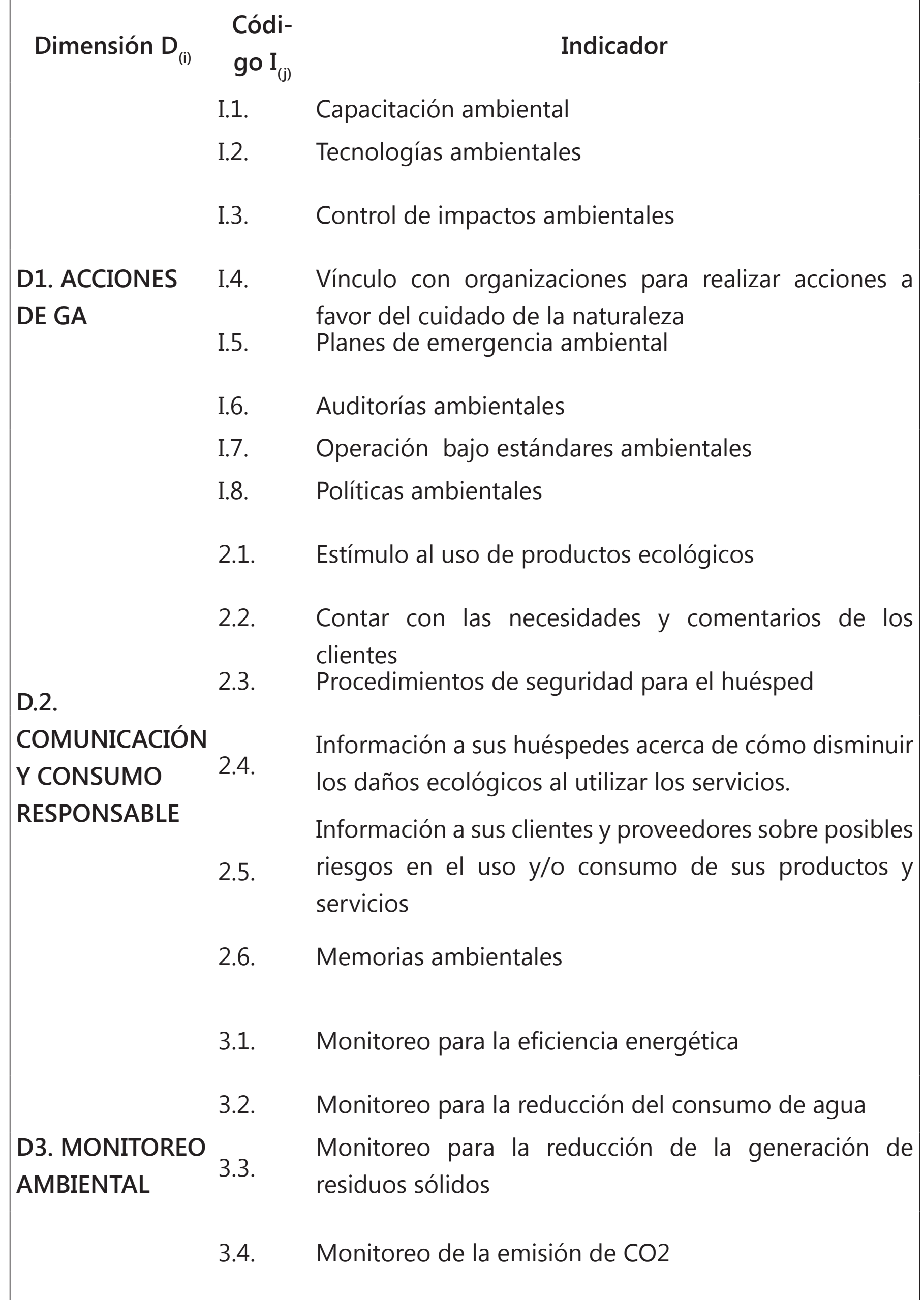


4.1. Conocimiento del SGA-RAN-Avala ambiental

4.2. Implantación de un SGA en la empresa

D4.

DISPOSICIÓN A

4.3. Intención de implantar un SGA en la empresa CUMPLIR

4.4. Reconocimiento de los beneficios del SGA

4.5. temática ambiental

4.6. Vínculo con el gobierno local

Fuente: Elaborada a partir de Vargas, Zizumbo y Viesca (2011, p. 137).

Para validar el cuestionario se seleccionaron siete expertos, tres profesores universitarios, dos consultores ambientales y dos especialistas del sector empresarial hotelero. La fiabilidad del cuestionario se determinó mediante el coeficiente de Alpha de Cronbach (0.815).

La evaluación se realiza en una escala nominal donde el criterio (+) significa que el indicador se aplica y el (-) que no se aplica. Posteriormente se determinaron los valores porcentuales (PRPD(i)) de las respuestas positivas (+) para cada (i) dimensión $(\mathrm{D}(\mathrm{i}))$ en el conjunto de los indicadores de los hoteles muestreados, así como el de respuestas positivas obtenidas en cada indicador (j) dentro de la muestra de cada país denominado PRPI(j).

$$
\operatorname{PRPD}(\mathrm{i})=\operatorname{CIPD}(\mathrm{i}) / \mathrm{NTH}
$$

\section{Donde;}

- CIPD (i) - Cantidad de indicadores con una evaluación positiva en la muestra de hoteles dentro de una (i) dimensión

- NTH - Número total de hoteles en la muestra

$\operatorname{PRPI}(j)=\frac{\operatorname{CRPI}(j)}{\mathrm{NTI}} * \mathrm{NTH}$

Donde;

- CRPI (j)- Cantidad de respuestas positivas para cada (j) indicador.

- NTI- Número total de indicadores evaluados en cada hotel

Para la valoración cualitativa de los resultados de la tipología de gestión 
ambiental se empleó la propuesta de Azqueta (2007) que ubica los hoteles en las categorías de "Líder", "Proactivo", "Reactivo", "Pasivo-indiferente" y "Negativo", para ello se construye una escala de intervalos mediante el método de amplitud y rango. El número de intervalos considerados (5) es la cantidad de categorías en las que se clasificará la gestión. El rango (R) es determinado según (3).

$$
R=(\operatorname{máx} P H)-(\operatorname{mín} P H)=100
$$

Donde:

- R- rango.

- $\mathrm{PH}$ - por ciento de aplicación del conjunto de indicadores en un hotel (valor máximo 100 y mínimo 0) según la escala.

La amplitud (C) de cada uno de los intervalos se determina según (4).

$C=R / K=100 / 5=20$

Donde:

- K- Es el número de categorías a considerar.

Según el valor de $C$, se establecen las escalas cualitativas que aparecen en la tabla 4. El valor PH es la media de los resultados obtenidos en el hotel en el conjunto de los ítems analizados.

Tabla 4. Escala de evaluación cualitativa para los valores alcanzados en el cuestionario

\begin{tabular}{|rl|}
\hline Intervalo & \multicolumn{1}{c|}{ Tipología para clasificar los hoteles } \\
$0 \leq \mathrm{PH}<20$ & Negativo \\
$20 \leq \mathrm{PH}<40$ & Pasivo-indiferente \\
$40 \leq \mathrm{PH}<60$ & Reactivo \\
$60 \leq \mathrm{PH}<80$ & Proactivo \\
$80 \leq \mathrm{PH}<100$ & Líder \\
\hline
\end{tabular}

Fuente: De los autores 2012

La información de los cuestionarios aplicados a las organizaciones, representadas por el coordinador ambiental, fue complementada con entrevistas a profundidad a otros directivos, que permitieron obtener información cualitativa adicional y corroborar las respuestas al cuestionario. 
Las hipótesis a demostrar son las siguientes:

HO: No existen diferencias significativas en cuanto a las dimensiones de gestión, indicadores y tipologías de gestión en los hoteles estudiados en Cancún, México y Varadero, Cuba.

$\mathrm{H} 1$ : Existen diferencias significativas en cuanto a las dimensiones de gestión, indicadores y tipologías de gestión en los hoteles estudiados en Cancún, México y Varadero, Cuba.

La muestra de cada destino se seleccionó de forma no probabilística, ya que se solicitó la participación del $100 \%$ de los hoteles con categoría 4* y 5* por ser los más representativos de ambos destinos y que integraron la población de estudio. Como resultado de este proceso se obtuvo la participación del 32.4\% de la población en el caso mexicano y $32.2 \%$ en el cubano. Las características comunes de la muestra en ambos destinos se aprecian en la Tabla 5.

Tabla 5. Características de las muestras seleccionadas en ambos países.

\begin{tabular}{|c|c|c|}
\hline Características & Cuba & México \\
\hline Turismo & Sol y playa & Sol y playa \\
\hline Modalidad & Todo incluido & Todo incluido \\
\hline Población de estudio & Hoteles $4^{*}$ y $5^{*}$ & Hoteles $4^{*}$ y $5^{*}$ \\
\hline Tamaño de la muestra & $\sim 32 \%$ & $\sim 32 \%$ \\
\hline $\begin{array}{l}\text { Existencia de un marco regulatorio de } \\
\text { referencia }\end{array}$ & Sí & Sí \\
\hline $\begin{array}{l}\text { Existencia de mecanismos nacionales de } \\
\text { certificación ambiental }\end{array}$ & Sí & Sí \\
\hline Participación en el estudio & Voluntaria & Voluntaria \\
\hline Antigüedad & 5-15 años & 5-15 años \\
\hline
\end{tabular}

Fuente: De los autores 2012

Para la comparación de los resultados se utilizaron gráficos de frecuencia, la prueba de Chi-cuadrada de Pearson y la prueba de Fischer para un 5\% $(p<0.05)$ 
para corroborar o no si las diferencias observadas eran significativas, en el último caso, es la prueba estadística de elección cuando la prueba de Chi-cuadrada no puede ser empleada por ser insuficiente el tamaño muestral.

Cabe mencionar que ambos estudios tuvieron una muestra inicial mayor que integraba hoteles con otras categorías, no obstante para efectos de la comparación de datos entre los dos destinos, se incluyeron solamente las empresas que presentaran una misma tipología de categorización.

\section{COMPORTAMIENTO ENTRE LOS RESULTADOS DE LA GESTIÓN AMBIENTAL EN CANCÚN Y VARADERO. COMPARACIÓN POR DIMENSIONES E INDICADORES}

De forma general existen similitudes y diferencias en los resultados de la gestión ambiental en el sector hotelero de los destinos de Cancún, México y Varadero, Cuba, si se atiende al comportamiento global de las cuatro dimensiones analizadas (Ver Figura 1).

Figura 1. Resultados globales de la evaluación expresados de acuerdo al cálculo para la muestra de cada destino PRPD(i).

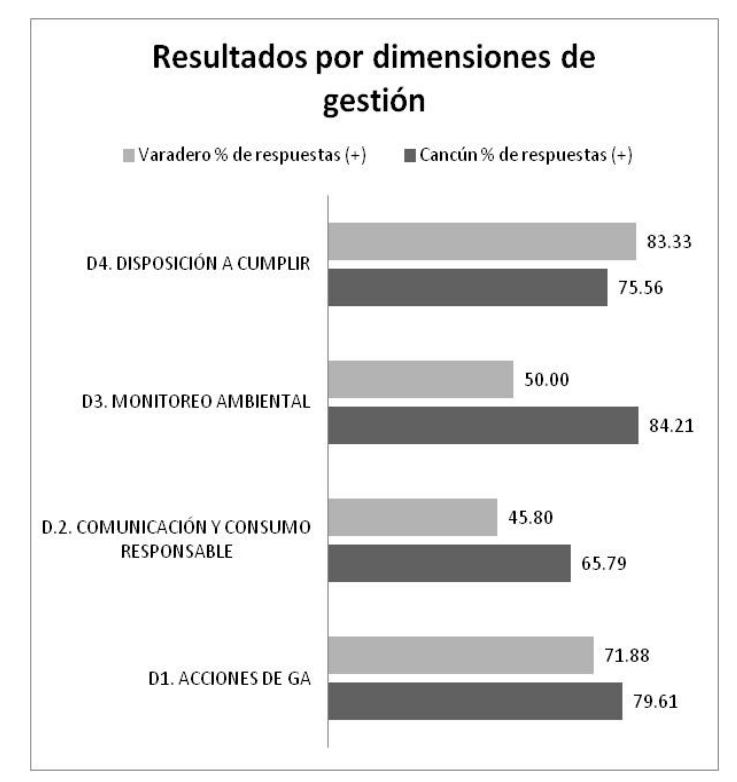

Fuente: Elaboración propia 
Se aprecia que ambos destinos tienen resultados similares en cuanto a la disposición a cumplir y las acciones de gestión ambiental, mientras en lo referido al monitoreo ambiental y la comunicación y consumo responsable los resultados son dispares (en ambos casos con mejores resultados en Cancún), aunque las mayores diferencias porcentuales se asocian al monitoreo ambiental.

Un análisis de la significación estadística de las diferencias en el comportamiento de las dimensiones consideradas se realizó mediante la prueba de Chi-cuadrada (Tabla 6.). El análisis aportó que a excepción de las acciones de gestión ambiental, donde la diferencia entre ambos destinos no es significativa, en el resto de las dimensiones sí lo son, así como en los resultados globales del estudio.

Tabla 6. Resultados de las pruebas de Chi-sq practicados a las respuestas relacionadas con el comportamiento de las cuatro dimensiones de análisis

\begin{tabular}{|c|c|c|c|c|c|c|}
\hline & Cancún & & Varadero & & Chi & Chi \\
\hline Dimensiones & $\begin{array}{l}\text { Respu- } \\
\text { estas (+) }\end{array}$ & $\begin{array}{l}\text { Respu- } \\
\text { estas (-) }\end{array}$ & $\begin{array}{l}\text { Respu- } \\
\text { estas }(+)\end{array}$ & $\begin{array}{l}\text { Respu- } \\
\text { estas (-) }\end{array}$ & $\begin{array}{l}\text { sq } \\
\text { calculado }\end{array}$ & sq Tabulado \\
\hline $\begin{array}{l}\text { D1. ACCIONES } \\
\text { DE GA }\end{array}$ & 121 & 31 & 69 & 27 & 1.962 & 3.89 \\
\hline D.2. & & & & & & \\
\hline $\begin{array}{l}\text { COMUNICACIÓN } \\
\text { Y CONSUMO } \\
\text { RESPONSABLE }\end{array}$ & 75 & 39 & 33 & 39 & 7.217 & 3.89 \\
\hline $\begin{array}{l}\text { D3. MONITOREO } \\
\text { AMBIENTAL }\end{array}$ & 64 & 12 & 24 & 24 & 16.711 & 3.84 \\
\hline $\begin{array}{l}\text { D4. DISPOSICIÓN } \\
\text { A CUMPLIR }\end{array}$ & 73 & 41 & 50 & 10 & 7.066 & 3.84 \\
\hline Total & 333 & 123 & 176 & 100 & 50.768 & 14.07 \\
\hline
\end{tabular}

Fuente: De los autores 2012

Un análisis más detallado en cuanto al comportamiento de los indicadores utilizados en cada dimensión se puede observar en la Figura 2. 
Figura 2. Resultados porcentuales positivos PRPI(j) alcanzados en cada uno de los indicadores incluidos en el cuestionario para los hoteles de cada destino.

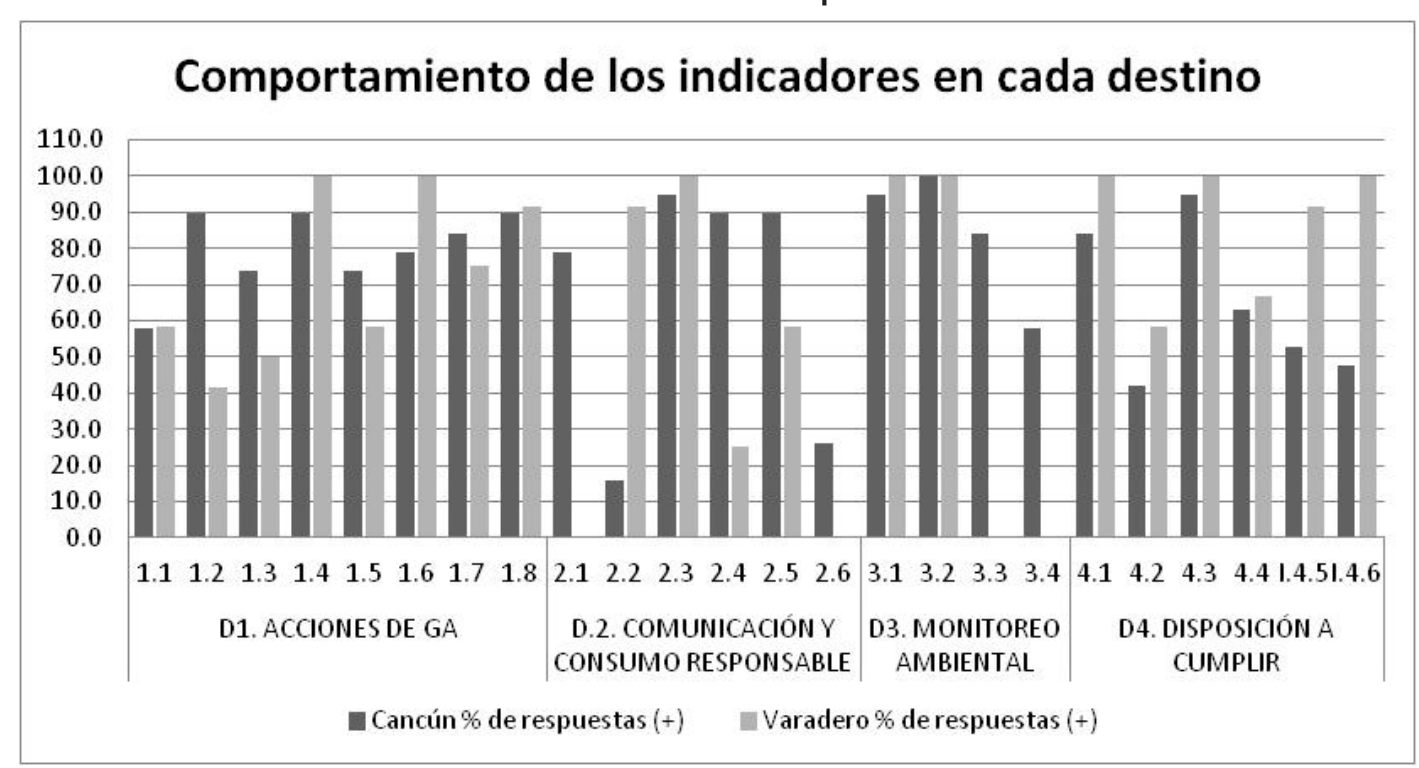

Fuente: Elaboración propia

Para profundizar en este análisis se aplicó la prueba de Chi-sq de Pearson y en los casos necesarios el test de Fischer $(P<0.05)$ para los resultados obtenidos en ambos destinos para cada indicador (Tabla7), lo cual arrojó que las diferencias estadísticamente significativas en el comportamiento de los indicadores se asocian a: la capacitación ambiental, el estímulo al uso de productos ecológicos, la Información a los huéspedes acerca de cómo disminuir los daños ecológicos al utilizar los servicios y sobre posibles riesgos ambientales, la publicación de memorias ambientales, así como al monitoreo de la generación de residuos sólidos y emisiones de $\mathrm{CO} 2$, en todos los casos con mejores resultados en el destino mexicano. Mientras que en los indicadores relacionados con la consideración de las necesidades y comentarios de los clientes, la implantación de modelos de gestión y los vínculos con el gobierno local, las diferencias favorecen el destino cubano. 
Tabla 7. Resultados de la prueba de Chi-sq para los indicadores evaluados.

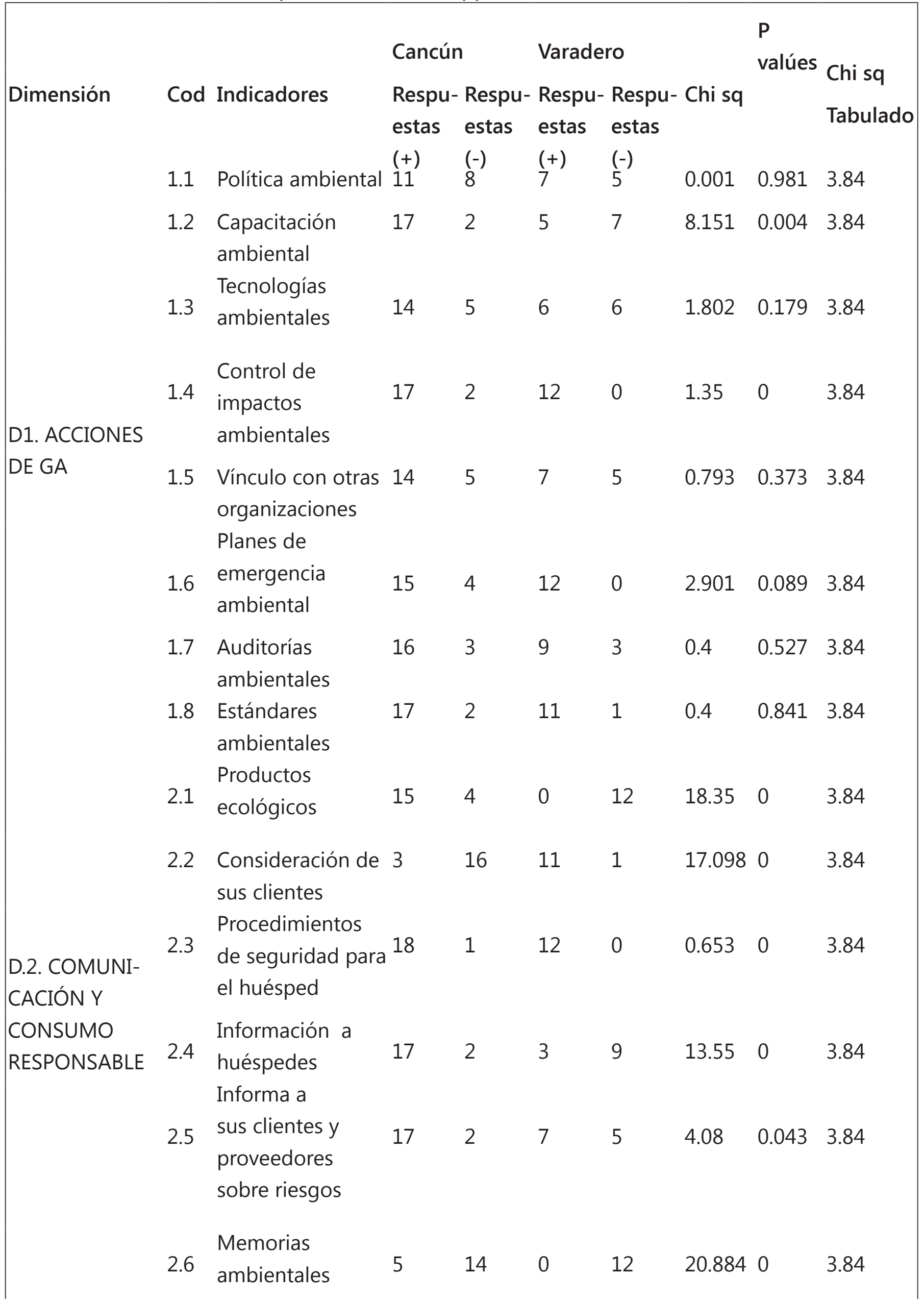




\begin{tabular}{|c|c|c|c|c|c|c|c|c|c|}
\hline \multirow{3}{*}{$\begin{array}{l}\text { D3. } \\
\text { MONITOREO } \\
\text { AMBIENTAL }\end{array}$} & 3.1 & $\begin{array}{l}\text { Monitoreo de } \\
\text { energía. }\end{array}$ & 18 & 1 & 12 & 0 & 0.653 & 0 & 3.84 \\
\hline & 3.2 & $\begin{array}{l}\text { Monitoreo de } \\
\text { agua. }\end{array}$ & 19 & 0 & 12 & 0 & 0.102 & 0 & 3.84 \\
\hline & 3.3 & $\begin{array}{l}\text { Monitoreo de } \\
\text { residuos sólidos }\end{array}$ & 16 & 3 & 0 & 12 & 20.884 & 0 & 3.84 \\
\hline \multirow{7}{*}{$\begin{array}{l}\text { D4. } \\
\text { DISPOSICIÓN A } \\
\text { CUMPLIR }\end{array}$} & 3.4 & $\begin{array}{l}\text { Monitoreo de la } \\
\text { emisión de CO2 }\end{array}$ & 11 & 8 & 0 & 12 & 10.768 & 0.001 & 3.84 \\
\hline & 4.1 & $\begin{array}{l}\text { Conoce el SGA- } \\
\text { PROFEPA } \\
\text { RAN-Aval } \\
\text { ambiental }\end{array}$ & 16 & 3 & 12 & 0 & 2.098 & 0.148 & 3.84 \\
\hline & 4.2 & $\begin{array}{l}\text { Implantación de } \\
\text { un modelo de } \\
\text { gestión }\end{array}$ & 8 & 11 & 7 & 5 & 10.768 & 0.001 & 3.84 \\
\hline & 4.3 & $\begin{array}{l}\text { Intención de } \\
\text { implantarlo }\end{array}$ & 18 & 1 & 12 & 0 & 2.098 & 0.148 & 3.84 \\
\hline & 4.4 & $\begin{array}{l}\text { Beneficios del } \\
\text { sistema de } \\
\text { gestión ambienta }\end{array}$ & 12 & 7 & 8 & 4 & 0.776 & 0.379 & 3.84 \\
\hline & 4.5 & $\begin{array}{l}\text { Apoyo de } \\
\text { organismos } \\
\text { públicos } \\
\text { especializados }\end{array}$ & 10 & 9 & 11 & 1 & 0.653 & 0 & 3.84 \\
\hline & 4.6 & $\begin{array}{l}\text { Vínculo con el } \\
\text { gobierno local }\end{array}$ & 9 & 10 & 12 & 0 & 9.323 & 0.002 & 3.84 \\
\hline
\end{tabular}

Fuente: Elaboración propia

\section{FORTALEZAS Y DEBILIDADES}

Para este análisis se consideraron aquellos indicadores que alcanzaron valores porcentuales de aplicación superiores al percentil 80 para las fortalezas, e inferiores al percentil 20 para las debilidades (figura 2).En Cancún la gestión ambiental de los hoteles se distingue por las fortalezas inherentes a la capacitación ambiental, el control de impactos ambientales, la realización de auditorías ambientales, la operación bajo estándares ambientales y los vínculos con otras 
organizaciones para acciones a favor del cuidado de la naturaleza. Resalta además por operar bajo estándares ambientales, contar con procedimientos de seguridad para el huésped e información al mismo acerca de cómo disminuir los daños ecológicos al utilizar los servicios del hotel y de los posibles riesgos ambientales relacionados. También constituyen elementos importantes dentro de la gestión ambiental de este destino el monitoreo ambiental del agua, la energía y los residuos, el conocimiento acerca del Programa Nacional de Auditoría Ambiental de PROFEPA y su intención de implantarlo. Por el contario, constituye el punto débil principal la toma en cuenta de las necesidades y comentarios de sus clientes respecto al desempeño ambiental del hotel como mecanismo de retroalimentación de la gestión.

En el caso de Varadero, constituyen fortalezas de la gestión ambiental de los hoteles estudiados el control de los impactos ambientales; operar bajo estándares ambientales y realizar auditorías ambientales. Resaltan además la elaboración de planes de emergencia ambiental, de procedimientos de seguridad para el huésped, así como tomar en cuenta las necesidades y comentarios de sus clientes respecto al desempeño ambiental del hotel. Otros resultados positivos se relacionan con el monitoreo de los consumos de agua y energía, el conocimiento acerca del RAN y el Aval ambiental y la intención de implementarlos, así como el apoyo recibido por los hoteles de organismos públicos especializados en la temática ambiental y los vínculos con las autoridades ambientales. En este destino constituyen deferencias en la gestión la no publicación de memorias ambientales, la ausencia de monitoreo de las emisiones de $\mathrm{CO} 2$ y residuos sólidos y la ausencia de un estímulo al consumo de productos ecológicos en las actividades del hotel.

\section{TIPOLOGÍA DE LOS HOTELES ACORDE A SU GESTIÓN AMBIENTAL}

La clasificación siguió los criterios de Azqueta et al. (2007) y mostró los resultados que se presentan en la figura 3 . 
Figura 3. Clasificación de los hoteles acorde a su gestión ambiental por ciento de aplicación del conjunto de indicadores en un hotel $(\mathrm{PH})$
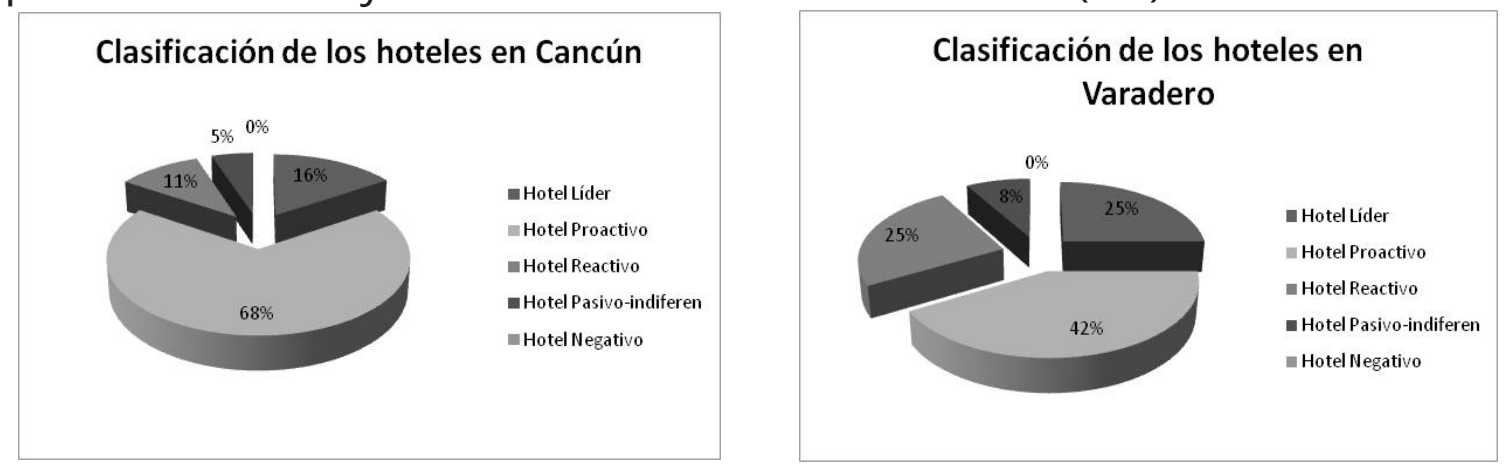

Fuente: Elaboración propia

Como se puede observar, en ambos destinos predominan los hoteles con una gestión favorable, es decir "Líder" o "Proactiva", ya que obtienen resultados positivos en más del $60 \%$ de los indicadores evaluados. Aunque debe señalarse que en Cancún representan el $84 \%$ de la muestra, mientras en Varadero son el 77\%, no obstante en Varadero hay más porcentaje de hoteles con gestión "Líder" con respecto a Cancún. La comparación entre categorías aplicando la prueba de Chi-sq de Pearson y en los casos necesarios el test de Fischer $(P<0.05)$ evidencia que en ninguna de las categorías se obtuvieron diferencias estadísticamente significativas (Tabla 8).

Tabla 8. Resultados de la prueba de Chi-sq respecto a la comparación de los grupos en ambos destinos.

\begin{tabular}{|llllll|}
\hline Categorías & Cancún & Varadero & \multicolumn{2}{c|}{ P } & \\
& Cantidad & Cantidad & Chi-sq & valúes & Chi-sq Tabulado \\
Hotel Líder & 3 & 3 & 0.4 & 0.527 & 3.84 \\
Hotel Proactivo & 13 & 5 & 2.162 & 0.141 & 3.84 \\
Hotel Reactivo & 2 & 3 & 1.39 & 0.286 & 3.84 \\
Hotel Pasivo-indiferente & 1 & 1 & 0.115 & 0 & 3.84 \\
Total & 19 & 12 & & & \\
\hline
\end{tabular}

Fuente: De los autores 2012 
La selección de las empresas hoteleras en las categorías de 4 y 5 estrellas como población de estudio se debió a que constituyen las de mayor peso en la planta hotelera de ambos destinos. Considerando que los cuestionarios evaluativos fueron entregados al $100 \%$ de la población de estudio, el por ciento de participación obtenido, tanto en Cancún como en Varadero, fue bajo, aproximadamente un $32 \%$ en ambos destinos. La muestra seleccionada no puede considerarse estadísticamente representativa, por ello los resultados obtenidos caracterizan solo las muestras estudiadas.

Los resultados demostraron la utilidad del cuestionario seleccionado para evaluar la gestión ambiental de las empresas hoteleras y de los destinos turísticos. En general se evidencia que la gestión ambiental de las muestras estudiadas en el sector hotelero en Cancún supera a la de Varadero en la dimensiones de: Comunicación y consumo responsable y monitoreo ambiental, mientras Varadero fue superior en la disposición a cumplir por parte del sector.

En el análisis particular entre el grupo de 24 indicadores evaluados mediante la prueba de Chi-sq se determinó cuáles conducían a diferencias estadísticamente representativas. Considerando igualmente el conjunto de indicadores se logró determinar las fortalezas y debilidades de la gestión ambiental en ambos destinos.

Finalmente, se clasificó la muestra acorde a los resultados de la gestión ambiental. En general existe correlación entre los resultados de ambos destinos, por lo que no se pueden considerar diferencias estadísticas entre los porcientos de hoteles integrados a las diferentes categorías en cada destino. No obstante, predominaron en Cancún los hoteles en la categoría de proactivos y en Varadero los de gestión reactiva y Varadero tuvo más hoteles líderes y menos con gestión pasiva-indiferente.

El resultado obtenido puede constituir una herramienta útil de benchmarking para el sector hotelero de ambos países, pero requiere de la sistematización futura del estudio y la ampliación de la muestra hasta valores estadísticamente representativos. 
ANSA. La hotelería verde en Europa una tendencia que nace. Disponible en: http://www. revistalideres.ec/tendencias/Acceso el 20 de sept. 2012.

ARUNDEL, A. y KEMP, R. Measuring Eco-innovation. England: United Nations University, 2009.

AZQUETA, D. et al. Introducción a la economía ambiental. Madrid: McGraw Hill, 2007.

Barómetro turístico de Cancún, 2012

BONILLA, M. J. y AVILÉS, C. Analysis of Environmental Statements Issued by EMAS Certified Spanish Hotels, Cornell Hospitality Quartely, 4, 2008, p. 381-394.

BUENO, E.; SALMADOR, M. P. y REYES, A. Influencia de los instrumentos voluntarios en la publicación de información ambiental de los hoteles españoles. Harvard Deusto Business Research,1(1), 2012, p. 37-51.

CABRERA, A. Medioambiente. Política de Desarrollo de Varadero. Cuba: Ministerio de Turismo, 2000.

CÉSPEDES, J. y DE BURGOS, J. La multidimensionalidad de la gestión ambiental de los servicios. Un análisis empírico de la industria hotelera. XI Congreso nacional ACEDME. Almería, 2002.

DE BURGOS, J. y CÉSPEDES, J. Un análisis del contenido de la gestión ambiental de los establecimientos hoteleros. Encuentro medioambiental almeriense: en busca de soluciones ambientales. Almería, 2003.

FONDO NACIONAL DE FOMENTO AL TURISMO FONATUR. (2010). Estadísticas de ocupación hotelera. Disponible en: http://datatur.sectur.gob.mx/work/docs/5 reporte semana/sem202010.pdf Acceso el 20 de marzo 2011.

GUZMÁN, A. La gestión ambiental en el sistema hotelero. Disponible en: http://www. abcformacion.com/contenidos/medio ambiente.htm. Acceso el 14 julio 2011.

INSTITUTO DE PLANEACIÓN Y DESARROLLO URBANO (IMPLAN). Plan estratégico de desarrollo sustentable. Benito Juárez, México: IMPLAN, 2007.

JÄNICEKE, M. Innovaciones para un uso sostenible de los recursos: reflexiones y propuestas. Ekonomiaz, 75, 2010, p. 71-84. 
KEMP, R. y PEARSON, P. Final report of the project 'Measuring Eco-innovation' (MEI) Disponible en: http://www.merit.unu.edu/MEI/index.php. Acceso el 14 sept.2012, 2008.

LÓPEZ, L. Tecnología para la Evaluación del Desempeño Ambiental de empresas hoteleras basada en índices de riesgo. Caso- Varadero. Tesis doctoral, Matanzas: Universidad de Matanzas "Camilo Cienfuegos", 2010.

LÓPEZ, L. Aplicación de un índice de riesgo para la Evaluación del Desempeño Ambiental de Empresas Hoteleras. Avanzada Científica, 1(14), 2011, p. 11-22.

LLULL, A.; ORFILA-SINTES, F.y FERNÁNDEZ, M. La proactividad de la estrategia medioambiental del sector hotelero: Una aplicación en Baleares, III Congreso de la Asociación HispanoPortuguesa de Economía de los Recursos Naturales y Ambientales, Palma, Mallorca, 2008.

MINISTERIO DEL TURISMO (MINTUR). Un millón de turistas en Varadero augura buena temporada. Disponible en: http://www.cubatravel.cu/otros/index.php?option=com_co ntent \&view $=$ article\&id=1012\%3Aun-millon- de-turistas-en-varadero-augura- buenatemporada\&lang=es. Acceso el 23 sept. 2011.

MINISTERIO DEL TURISMO (MINTUR). Datos sobre las empresas hoteleras cubanas Disponible en: www.cubatravel.cu. Acceso el 23 sept. 2012.

MIRET-PASTOR, L.; DEL-VAL, M. y PEIRÓ, A. ¿Cómo medimos la Eco-innovación? Análisis de indicadores en el Sector Turístico. Tec. Empresarial, 5(2), 2011, p.15-25.

MOLINA, J.F., CLAVER, E.; PEREIRA, J.; TARÍ, J.J. Gestión de la calidad y medioambiental y rendimiento empresarial: un análisis de los hoteles españoles. Revista Europea de Dirección y Economía de la Empresa, 2(18), 2009, p.63-78.

MOYA, B.; CABRERA, A.; CASTILLO, L.yROJO, J.Varadero ante el cambio global medioambiental. En SÁNCHEZ, R. y BONILLA, A. (edt). Urbanización, Cambios Globales en el Ambiente Desarrollo Sustentable en América Latina. Brasil: IAI, INE, UNEP. P.33-59,2007.

NC-ISO 14031. Gestión ambiental. Evaluación del desempeño ambiental, directrices. Norma Cubana. Vig. 2005.

PAZ, S. Tendencias Mundiales del Turismo: La zona del Caribe, Foro de análisis: Cancún: EI auge y la crisis. Cancún, 1995.

PELEGRÍN, A.; ARACELI, M.; ELOINA, S. Modelo de costos ambientales para empresas turísticas. Experiencias en Cuba y México. Foro Ambiental de Contabilidad ambiental y Social. Argentina, 2010. 
PONCE, H. G. y LABAT, B. N. La información medioambiental de las empresas análisis de la regulación existente: Un estudio empírico sobre la información medioambiental que suministran las empresas incluidas en el índice bursátil IBEX-35. 1996-1999, IX Reunión de Economía Mundial, Madrid, 2007.

PROCURADURÍA FEDERAL DE PROTECCIÓN AL AMBIENTE. Término de Referencia para la realización de auditorías ambientales. Sub-procuraduría de Auditoría ambiental. México. Disponible en: http://www.profepa.gob.mx/Acceso el 23 sept. 2012, 2009.

PROCURADURÍA FEDERAL DE PROTECCIÓN AL AMBIENTE. Datos sobre la certificación ambiental del sector hotelero. Disponible en: http://www.profepa.gob.mx/Acceso el 23 sept. 2012, 2010.

RIVAS, L.; CABALLERO, V. e IZQUIERDO, M. Evaluación de la Cuenca de Mono II para su empleo en el vertimiento de arena en la playa de Varadero. Serie Oceanológica, 6, 2009, p. 27-38.

ROMERO, R. Política municipal y desarrollo urbano de un modelo turístico. Cancún: 19752002. En: MACÍAS, C. y PÉREZ, R. (Coords.) Cancún los avatares de una marca turística global, p.15-141, 2009.

SALES, F.V.; ACEVEDO, M. L.; ALENCAR, S.; REBOUGAS, S. y OLIVEIRA, E. ¿Prefieren los turistas hospedarse en hoteles cuyos restaurantes adoptan prácticas de sustentabilidad? Estudios y Perspectivas de Turismo, 21, 2010, p.1159-1175.

SILENIS, A., HERNÁNDEZ, R., SILVESTRE, K., \& GÓMEZ, O. Análisis de los elementos que conforman la responsabilidad social corporativa. Formación Gerencial, 2, 2008, p.55-62.

TERRY, C. Producción más limpia en el sector turístico. Cub@: Medio Ambiente y desarrollo, 2(3), 2002, p. 1- 4.

VARGAS, E.E.;ZIZUMBO, L.y VIESCA, C. Turismoy ambiente. Unestudio delos comportamientos ambientales en el sector hotelero de Cancún. En: JUÁREZ, D. (edit.). Turismo, economía y medio ambiente. Madrid: Editorial Académica Española, p. 123-147, 2011. 